\title{
Upper limb kinematics: Development and reliability of a clinical protocol for children
}

\author{
Ellen Jaspers ${ }^{\mathrm{a}, *}$, Hilde Feys ${ }^{\mathrm{a}}$, Herman Bruyninckx ${ }^{\mathrm{b}}$, Jaap Harlaar ${ }^{\mathrm{c}}$, Guy Molenaers ${ }^{\mathrm{d}, \mathrm{e}}$, Kaat Desloovere ${ }^{\mathrm{a}, \mathrm{e}}$ \\ a Department of Rehabilitation Sciences, Faculty of Kinesiology and Rehabilitation Sciences, Katholieke Universiteit Leuven, Belgium \\ ${ }^{\mathrm{b}}$ Department of Mechanical Engineering, Faculty of Engineering, Katholieke Universiteit Leuven, Belgium \\ ${ }^{c}$ Department of Rehabilitation Medicine, Research Institute MOVE, VU University Medical Center, Amsterdam, The Netherlands \\ ${ }^{\mathrm{d}}$ Department of Musculoskeletal Sciences, Faculty of Kinesiology and Rehabilitation Sciences, Katholieke Universiteit Leuven, Belgium \\ ${ }^{\mathrm{e}}$ Clinical Motion Analysis Laboratory, CERM, University Hospital Pellenberg, Belgium
}

\section{A R T I C L E I N F O}

Article history:

Received 18 June 2010

Received in revised form 18 November 2010 Accepted 28 November 2010

\section{Keywords:}

Upper limb

Kinematics

Reliability

Children

\begin{abstract}
A B S T R A C T
This study proposed a child-friendly measurement procedure for the three-dimensional analysis of upper limb movements, based on a comprehensive movement protocol. Within and between session reliability was tested in a group of 10 typically developing children (TDC) (mean age $10.3 \pm 3.2$ years). The movement protocol was constructed for children with hemiplegic cerebral palsy (HCP) and contained three reach tasks (forwards, upwards, sideways), three reach-to-grasp tasks (with objects requiring different hand orientations) and three gross motor tasks. Upper limb kinematics were calculated following the ISBguidelines. Reliability of movement duration/speed and endpoint joint angles was assessed with the intraclass correlation coefficient; similarity of the waveforms with the coefficient of multiple correlation; measurement errors were also calculated.

Reliability coefficients were generally high for movement duration/speed and most kinematic parameters. Endpoint angles for scapular tilting, shoulder elevation plane and elevation, elbow flexionextension and wrist ulnar-radial deviation showed highest reliability. Angular waveforms were best repeated for scapular medio-lateral rotation and pro-retraction, shoulder elevation plane and elevation, and elbow flexion-extension. Results also seemed task-dependent.

This study indicated that the proposed procedure could be used reliably to quantify upper limb movements in TDC. However, to compose proper age-related standards for the different tasks, larger study samples are needed. This will also help with a well-founded task-selection depending on the joints of interest. Finally, further research will need to establish the reliability in children with HCP.
\end{abstract}

(c) 2010 Elsevier B.V. All rights reserved.

\section{Introduction}

There seems an emerging interest among researchers and clinicians to use three-dimensional movement analysis (3DMA) to assess upper limb (UL) movements in children [1-3]. Such objective data may provide more sensitive information on UL movement patterns compared to clinical evaluations, thereby supporting treatment planning for children with hemiplegic cerebral palsy (HCP). Clinical application of an UL 3D-measurement firstly requires the establishment of the biomechanical model and secondly of a set of relevant tasks [4].

Although several biomechanical models have been proposed, they vastly vary in complexity, number of segments, joint degrees of freedom (DoF), and marker configurations [5-8]. To standardize the reporting of UL kinematics, the International Society of

\footnotetext{
* Corresponding author. Tel.: +32 16 329117; fax: +32 16329197

E-mail address: ellen.jaspers@faber.kuleuven.be (E. Jaspers).
}

Biomechanics (ISB) published recommendations on the definition of joint coordinate systems and rotation sequences [9]. Nonetheless, most studies on UL kinematics in typically developing children (TDC) [1,10] and children with HCP [3] have not yet incorporated these guidelines. Moreover, there is no general consensus on which tasks should be assessed [3]. Given the variety in UL functions, it is however crucial to design a comprehensive movement protocol containing a set of clinically relevant tasks.

Once the biomechanical model and movement protocol have been designed, the reliability of the measurement procedure needs to be established in TDC. Thus far, merely two studies have assessed the reliability of UL kinematics in TDC [11,12]. While both studies reported good within and between session reliability, only Butler et al. [11] provided an estimate of the measurement error. Still, information on the measurement errors is indispensable for a proper interpretation of reliability results. Furthermore, these studies used a variation of the ISB-guidelines and scapular movements were not assessed [11,12]. Given the role of the scapula in the normal movement of the humerus [13], it should 
however be taken into account when evaluating UL motor performance.

This study proposes a child-friendly and clinically feasible 3DMA for the objective evaluation of UL movements, maximizing the DoF and employing a comprehensive movement protocol. A set of tasks was selected to represent functional and clinically relevant UL activities for children with HCP. Within and between session reliability and the measurement error of temporal and kinematic parameters were assessed in a group of TDC.

\section{Methods}

\subsection{Participants}

Ten TDC ( 6 boys, 4 girls) - mean age 10.3 ( \pm 3.2 years), ranging from 6.0 to 14.6 years - voluntarily participated. Children had no history of musculoskeletal or neurological problems. Nine children were right-handed and one left-handed. Ethical approval was granted by the Ethics Committee of the University Hospital Leuven (Belgium). Written informed consent was obtained from all children's parents.

\subsection{Movement protocol}

The movement protocol was constructed based on a literature review [3], discussions with experts in the field of HCP, and tasks comprised in the Melbourne Assessment [14]. Nine tasks were selected to reflect the UL movement deficits of the hemiplegic child. Tasks were subdivided into three groups: reaching, reach-to-grasp, and gross motor functions. The reach tasks were executed in different directions: forwards $(R F)$, upwards $(R U)$ and sideways $(R S)$. The reach-to-grasp tasks required different hand orientations: either forearm pronation, i.e. grasp a ball (spherical grasp, $R G S$ ), a horizontally oriented cylinder (horizontal grasp, $R G H$ ), or forearm supination, i.e. grasp a vertically oriented cylinder (vertical grasp, $R G V$ ). The gross motor tasks included hand to mouth (HTM), hand to top of head $(H T H)$ and hand to contralateral shoulder (HTS), representing common daily activities, e.g. eating, grooming and dressing.

Reach forwards, sideways and the three reach-to-grasp tasks were all executed at shoulder height. The height for upward reaching was taken at eye-height.

\subsection{Kinematic model}

The kinematic model followed the implementation described by van Andel et al. [15], marker configurations were adapted for children (Fig. 1). Five segments were included (trunk, scapula, humerus, forearm, hand) and four joints were considered (scapulathoracic (scapula), humerothoracic (shoulder), elbow, wrist), as well as trunk kinematics. Anatomical coordinate systems (ACS) and joint rotation sequences were defined according to the ISB-guidelines [9].

\subsection{Set-up}

Every child was evaluated on two occasions, 2-10 days apart, at the clinical motion analysis laboratory (University Hospital Pellenberg, Belgium), by the same assessor. Seventeen markers, clustered on tripods and cuffs, were placed over the child's arm and trunk (Fig. 1). Due to the non-cyclic nature of UL movements, a custom-made chair with adjustable foot and back support and reaching distance and height was developed (Fig. 1). The reference position was $90^{\circ}$ flexion in hip and knees, with the low back supported and the hands placed on the ipsilateral knee. Reaching distance and height were based on the arm length (distance between acromion and third metacarpophalangeal joint with the arm hanging down) and shoulder height in sitting position, respectively. To maximize the standardization of the test set-up, the child's sitting position, and reaching distance and height were carefully noted and reapplied the second test session. While seated in the chair, static trials were collected to record the reference position and to digitize the anatomical landmarks within their respective marker cluster [17], using a pointer with four linear markers [15]. Palpation was done based on precise definitions of palpable landmarks [19].

After these static trials, children completed the selected tasks with the nondominant arm (non-preferred), at self-selected speed. The non-dominant arm was chosen to allow future comparison with the hemiplegic arm (non-preferred) in children with HCP [3]. The hand on the ipsilateral knee - indicated with rough tape was the start and end position of every task. Before every trial, children were instructed to sit up-straight, the desired task was demonstrated by the therapist and children were given a practice trial. Each task was repeated four times during one single movement trial, resulting in four repeats (movement cycles) per trial. Three successful trials for every task were collected, producing 12 movement cycles per task.

3D-marker tracking was done with 12 infrared Vicon-cameras sampling at $100 \mathrm{~Hz}$ (Vicon Motion Analysis system, Oxford Metrics, UK), and filtered using spline-interpolation [20].

\subsection{Data analysis}

Movement cycles were visually identified with the stop-frame feature and frame-by-frame inspection of the moving markers. One movement cycle was

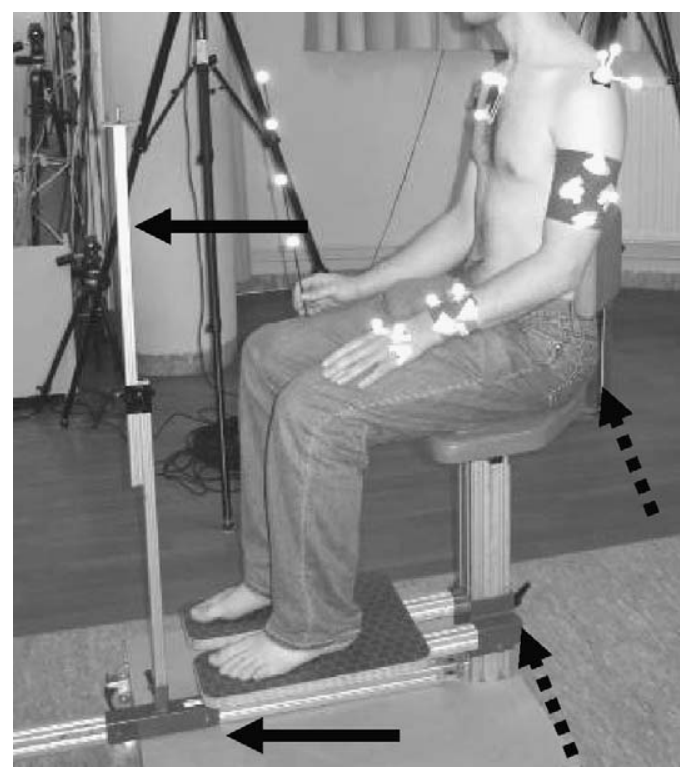

Fig. 1. (1) Marker placement: every segment is completely defined in space by a cluster of at least three non-collinear markers, with a total of 17 markers (14 mm diameter). The marker clusters were used to calculate the orientation of the segmental technical coordinate systems (TCS) by means of singular value decompositions [16]. Anatomical landmarks (ALs) were palpated and digitized during static trials, using a pointer with four linear markers. The ALs were defined within their respective segmental TCS [17], and subsequently used to construct the segmental anatomical coordinate systems (ACS). Elbow and wrist joint centers were calculated based on the concurrent ALs. The shoulder rotation centre was estimated based on a linear regression equation [18]. (2) Every child was seated in the custommade chair with adjustable foot and back support (dotted arrows) and reaching distance and height (full arrows).

defined from 'hand on ipsilateral knee' to the 'point of task achievement' (PTA), i.e. the instant when the range of movement (ROM) needed for successful task execution was achieved [10]. Only the second and third cycle of every trial were retained for further data processing - as these trials were not corrupted by potential start/stop strategies of the child - resulting in a total of six movement cycles per task.

Further data processing was done with Matlab ${ }^{\mathbb{R}}$ (BodyMech, http://www.bodymech.nl). The interface was adjusted to allow processing of c3d-data and the required routines were added to calculate UL kinematics [15]. Movement cycles were time-normalized (0-100\%) and each joint angle was visualized as a function of time. Erroneous signals due to artefacts caused by marker occlusion were not included in the statistical analysis. Temporal parameters (movement duration, speed) and joint angles at PTA were derived for every movement cycle of every task.

\subsection{Statistical analysis}

Within session reliability was based on the comparison of six movement cycles in the first session and gives an indication of the consistency of task performance of each child. Between session reliability was based on the comparison of the performance between both test sessions, and gives an indication of the errors introduced when the measurement is repeated over time.

Reliability of the temporal parameters and joint angles at PTA was assessed with the intraclass correlation coefficient (ICC), with 95\% CI. The standard error of measurement (SEM) was estimated from the square root of the mean square error term from the two-way ANOVA [21]. Within session reliability was calculated with $\operatorname{ICC}_{\mathrm{w}}(2,1)$ and $\mathrm{SEM}_{\mathrm{w}}$, based on single data; between session reliability with $\operatorname{ICC}_{\mathrm{b}}(2, \mathrm{k})$ and $\mathrm{SEM}_{\mathrm{b}}$, using averaged data [21]. ICC-values $>0.80$ represent very high, 0.60 0.79 moderately high, $0.40-0.59$ moderate and $<0.40$ low reliability [22].

Similarity of the angular waveforms was evaluated with the adjusted coefficient of multiple correlation (CMC) [23]. CMCs were computed for every child and median values were then calculated. CMC-values $>0.90$ were considered excellent, $0.80-0.89$ good, $0.60-0.79$ moderate and $<0.60$ poor. Similar to the SEM, the measurement error was also calculated for the waveforms $(\sigma)$ [24]. Within session errors $\left(\sigma_{\mathrm{w}}\right)$ were based on the inter-trial comparison of the waveforms and reflect the intrinsic reliability of UL kinematics. Between session errors $\left(\sigma_{\mathrm{b}}\right)$ were calculated based on the inter-session comparison of all waveforms and reflect the extrinsic errors arising from various methodological sources. Within session errors were used as a reference level to which the extrinsic sources of error can be compared, and reported as the ratio of between to within session error [24]. 

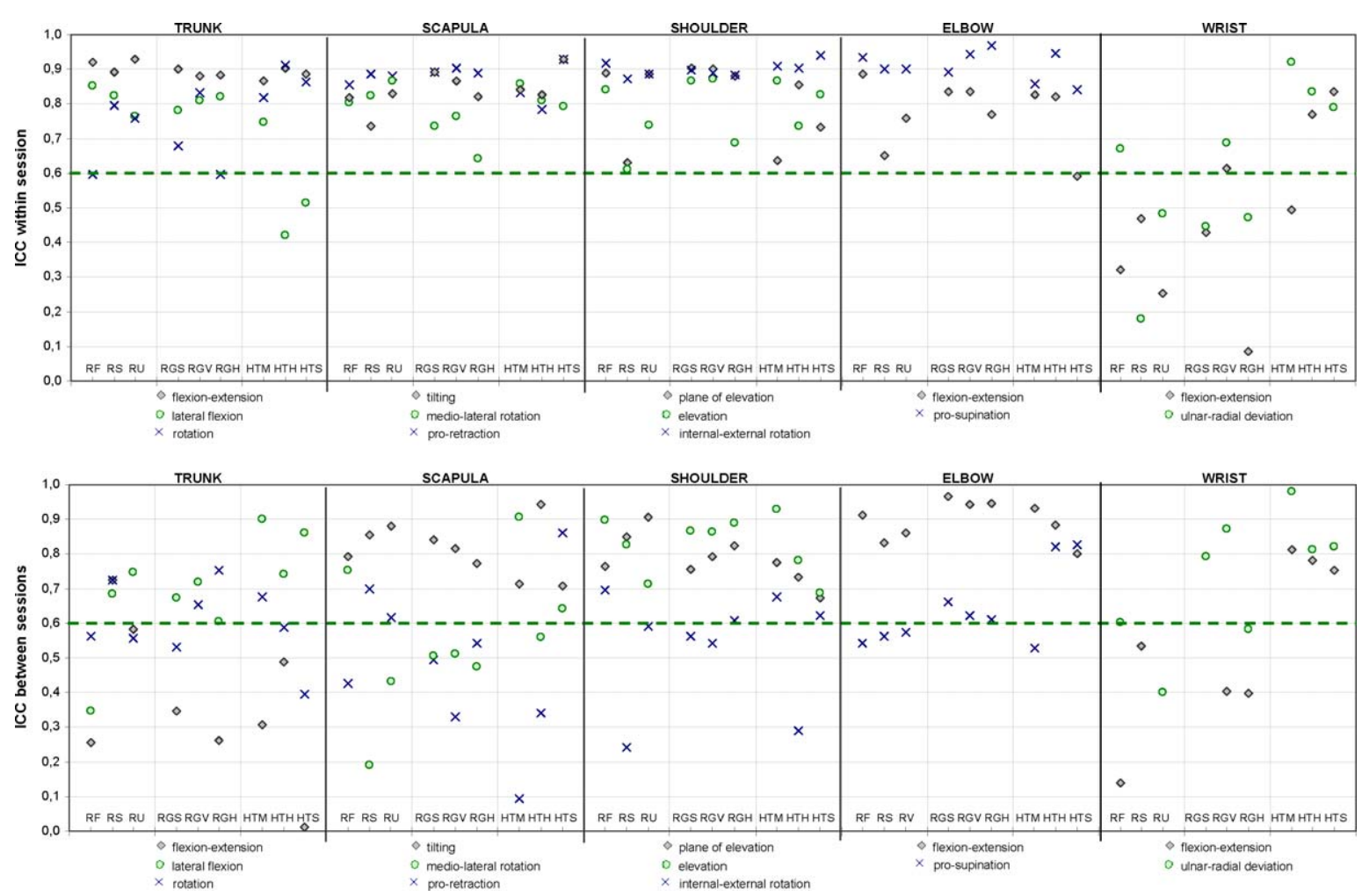

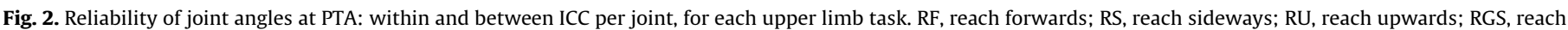
grasp spherically; RGV, reach grasp vertically; RGH, reach grasp horizontally; HTM, hand to mouth; HTH, hand to head; HTS, hand to shoulder.

\section{Results}

Information on within and between reliability coefficients can be found in Figs. 2 and 3. Full details are available as Supplementary Material. Within and between measurement errors are given in Tables 1 and 2 .

\subsection{Temporal parameters}

Moderately high to very high within and between session ICCs were found for movement duration and speed for all tasks (ICC $0.62-0.96)$. SEM ranged between $0.04-0.10 \mathrm{~s}$ and $0.02-0.07 \mathrm{~m} / \mathrm{s}$, for duration and speed, respectively.

\subsection{Joint angles at PTA}

Within session ICCs were moderately high to very high for all joint angles at PTA (ICC > 0.60), except for the wrist. Between session ICCs for the scapula, shoulder and elbow angles at PTA were also exceeding 0.60 , though lower values were found for axial rotations. Wrist and trunk between ICCs were generally lower. Within SEM-values for the trunk, scapula and shoulder and for elbow pro-supination were $<5^{\circ}$ for all tasks, except shoulder elevation plane for HTM and HTH and shoulder rotation for $\mathrm{HTH}\left(\mathrm{SEM}_{\mathrm{w}} 5-6^{\circ}\right) . \mathrm{SEM}_{\mathrm{w}}$ for elbow flexion ranged from $2.3^{\circ}$ to $4.9^{\circ}$ for $\mathrm{RU}, \mathrm{RGV}$ and the gross motor tasks, and between $5.3^{\circ}$ and $6.1^{\circ}$ for the other tasks. At the level of the wrist, $\mathrm{SEM}_{\mathrm{w}}$ ranged from $4.1^{\circ}$ to $8.5^{\circ}$, with lowest values for ulnar-radial deviations. Between SEM-values were slightly higher, though the majority of values was $<7^{\circ}$. Only shoulder rotation and elbow prosupination had $\mathrm{SEM}_{\mathrm{b}}$ ranging from $7^{\circ}$ to $9^{\circ}$ for all tasks.

\subsection{Angular waveforms}

Within and between session reliability was highest for scapula and shoulder waveforms during reaching and reach-to-grasp, for elbow flexion during all tasks, and for pro-supination during the gross motor tasks and $\mathrm{RGV}\left(\mathrm{CMC}_{\mathrm{w}}>0.80\right)$. Trunk and wrist waveforms had lower CMCs. Lower between session CMCs were also found for joint waveforms in the transverse plane (scapula pro-retraction, shoulder rotation, elbow pro-supination). Between session waveform errors ranged from $1.6^{\circ}$ to $9.7^{\circ}$. Errors were lowest for trunk and scapula $\left(\sigma_{\mathrm{b}}<5^{\circ}\right)$, followed by shoulder elevations and wrist ulnar-radial deviations $\left(\sigma_{\mathrm{b}} 5-7^{\circ}\right)$. Highest errors were found for shoulder rotations $\left(\sigma_{\mathrm{b}}>8^{\circ}\right)$. At the level of the elbow, lowest errors were found for flexion-extension during the gross motor tasks and for pro-supination during the reach-tograsp tasks $\left(\sigma_{\mathrm{b}} 5-7^{\circ}\right)$. Error-ratios ranged from 1.0 to 2.0 for shoulder, scapula and wrist angles, as well as trunk lateral flexion and elbow flexion-extension. Elbow pro-supination had good error-ratios only for those tasks requiring forearm supination $(r<1.5)$.

\section{Discussion}

This study proposed a child-friendly measurement procedure to objectively quantify UL movements, based on a comprehensive movement protocol. The reliability of movement duration/speed, joint angles at PTA and angular waveforms was assessed in 10 TDC.

Results indicated good reliability for movement duration/speed for all tasks. Within session ICCs were generally high for all joint angles at PTA for the different tasks, indicating good endpoint reliability. Between session reliability was highest for scapula, shoulder and elbow kinematics, while wrist and trunk kinematics seemed less reliable. However, inspection of the wrist angles at PTA showed that within-subject variability was generally low, while between-subject variability differed among the tasks. As it is well known that the magnitude of the ICC is vastly influenced by the amount of between-subject variability [21], the lower ICCs at the level of the wrist should be interpreted with this in mind. Furthermore, these lower ICCs contrast with the good SEM-values 

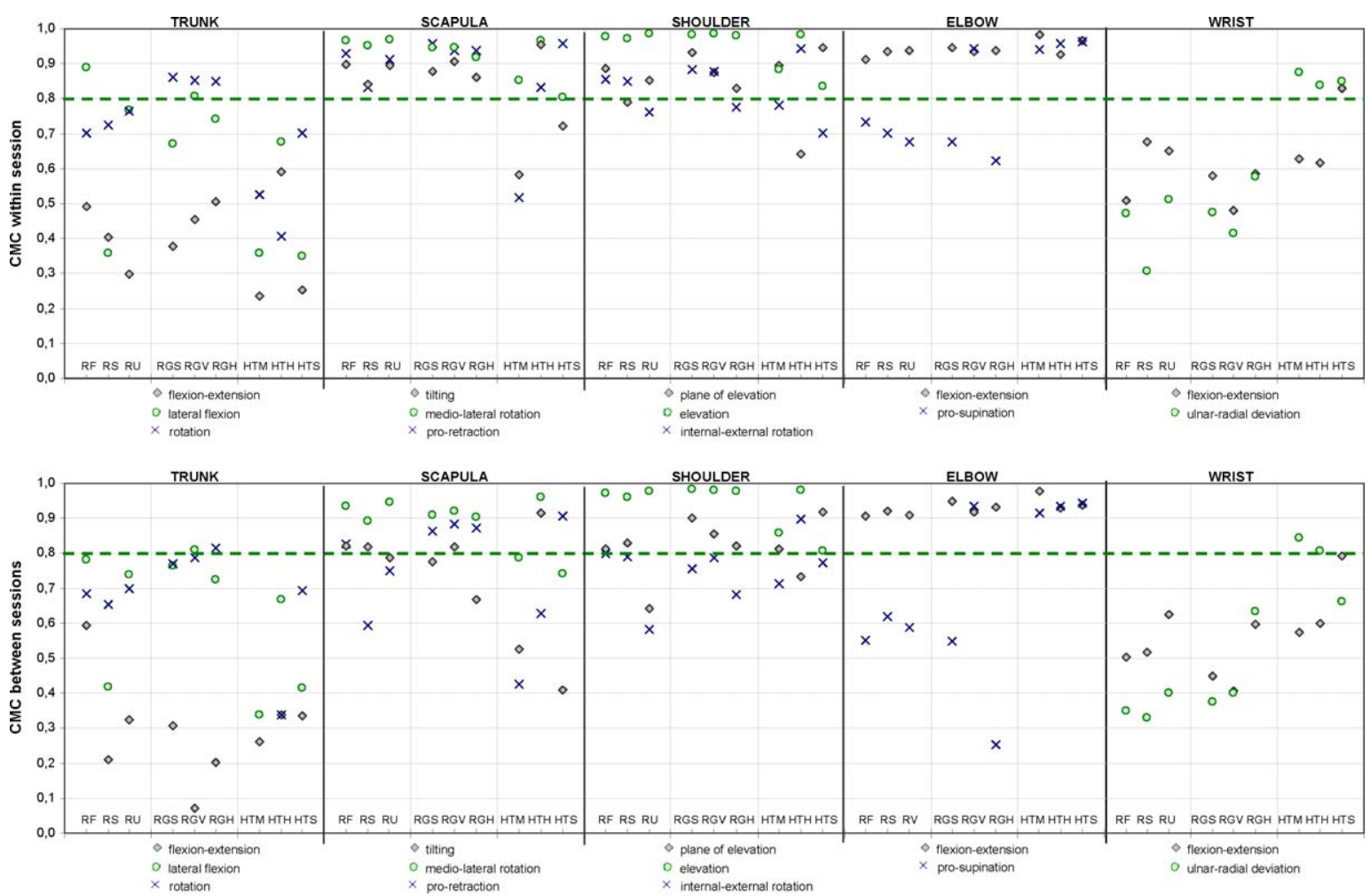

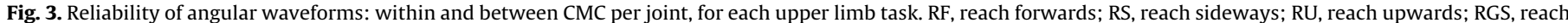
grasp spherically; RGV, reach grasp vertically; RGH, reach grasp horizontally; HTM, hand to mouth; HTH, hand to head; HTS, hand to shoulder

that were found for wrist flexion $\left(2-8^{\circ}\right)$ and deviation $\left(2-5^{\circ}\right)$ for all tasks, thereby supporting the reliability at the level of the wrist. Lower between session ICCs were also found for shoulder axial rotation, scapular pro-retraction and medio-lateral rotation at PTA. Given their good within session reliability, methodological sources of error should be considered when measuring these joint angles, e.g. marker placement, palpation errors and joint center calculation [24].

Waveform similarity was assessed with the CMC and good to excellent values were found for most joints during the different tasks. Trunk and wrist kinematics had markedly lower within and between CMCs, suggesting a large variability in task execution. For the wrist, this was confirmed by visual inspection of the individual waveforms. Trunk angles were calculated relative to the lab coordinate frame, whereby the reference position of the child was used as the "zero-position". Since children were not strapped onto the chair, variations in the starting position already introduced some differences between trials. This variability might be reduced by restricting trunk movements. However, a significant increase of trunk compensatory movements has already been reported in children with HCP [3], and valuable information will be lost when restraining the trunk.

Joint movements with a large ROM typically show high CMCs, whilst joint movements with less ROM result in lower CMCs [25]. In the current study, this effect can be seen for elbow prosupination during tasks that did not require forearm movement versus tasks involving forearm rotation. Adding tasks to the movement protocol that specifically require wrist movements might help to improve reliability of this joint. Reid et al. [12] found similar, though somewhat lower, CMCs for shoulder flexion/ abduction, elbow and wrist movements in 10 TDC during RF, RS, HTM and a pro-supination task. The higher CMCs reported in our study might partly be ascribed to the thorough standardization.

Differences in measured joint kinematics arise from natural variation in task execution, but also from experimental errors.
While natural variation can only be measured and managed, experimental errors are dependent on the quality of the measurement and may arise from various methodological sources (palpation inaccuracies, marker placement, joint center calculation) [24]. The current study focused on inter-trial and intersession errors. Knowledge on the magnitude of these errors is imperative to distinguish clinically meaningful changes in intervention and longitudinal studies. This will help determining which participants show a 'genuine' improvement, rather than a difference in performance due the measurement error. Between session SEM of the joint angles at PTA ranged from $1.4^{\circ}$ to $9.2^{\circ}$ for the reach tasks, from $1.9^{\circ}$ to $8.6^{\circ}$ for the reach-to-grasp tasks and from $1.0^{\circ}$ to $9.9^{\circ}$ for the gross motor tasks, with highest errors found for shoulder rotation and elbow pro-supination. Measurement errors of the waveforms ranged from $1.7^{\circ}$ to $9.7^{\circ}, 1.7^{\circ}$ to $8.6^{\circ}$, and $1.6^{\circ}$ to $8.5^{\circ}$ for the reach, reach-to-grasp, and gross motor tasks respectively. These values are slightly higher than reported by Butler et al. [11] in seven TDC (between session error 1.6-4.8 ${ }^{\circ}$ ).

When interpreting results from the current study, some assumptions and limitations should be considered. Local coordinate systems and rotation sequences were defined according to the ISB-recommendations [9]. For the description of shoulder kinematics, the rotation order YXY implies the possible occurrence of gimbal lock at the poles, i.e. $0^{\circ}$ and $180^{\circ}$ of elevation [26]. Values near the gimbal lock positions should be used with caution due to the bad numerical conditioning of the Euler angle calculation near those singular positions. For this study, start and end position was defined as 'hand on the ipsilateral knee'. This position ensured a minor shoulder elevation at all times to reduce the disturbances of a near gimbal lock position and results showed that this sequence is appropriate for the selected tasks. Further study will need to clarify the rationale and clinical utility of other movement dependent rotation sequences for the shoulder, e.g. 'flexionabduction-rotation', 'abduction-flexion-rotation' [27], or 'conjunct rotation' [28]. 


\begin{tabular}{|c|c|c|c|c|c|c|c|c|c|c|c|c|c|c|c|c|c|c|}
\hline & \multicolumn{2}{|l|}{ Reach forwards } & \multicolumn{2}{|l|}{ Reach sideways } & \multicolumn{2}{|l|}{ Reach upwards } & \multicolumn{2}{|l|}{$\begin{array}{l}\text { Reach to grasp } \\
\text { spherically }\end{array}$} & \multicolumn{2}{|l|}{$\begin{array}{l}\text { Reach to grasp } \\
\text { vertically }\end{array}$} & \multicolumn{2}{|l|}{$\begin{array}{l}\text { Reach to grasp } \\
\text { horizontally }\end{array}$} & \multicolumn{2}{|l|}{ Hand to mouth } & \multicolumn{2}{|l|}{ Hand to head } & \multicolumn{2}{|c|}{ Hand to shoulder } \\
\hline & $\begin{array}{l}\text { Mean } \\
\text { (SD) }\end{array}$ & $\mathrm{SEM}_{\mathrm{w}}$ & $\begin{array}{l}\text { Mean } \\
\text { (SD) }\end{array}$ & $\mathrm{SEM}_{\mathrm{w}}$ & $\begin{array}{l}\text { Mean } \\
(\mathrm{SD})\end{array}$ & $\mathrm{SEM}_{\mathrm{w}}$ & $\begin{array}{l}\text { Mean } \\
\text { (SD) }\end{array}$ & $\mathrm{SEM}_{\mathrm{w}}$ & $\begin{array}{l}\text { Mean } \\
\text { (SD) }\end{array}$ & $\mathrm{SEM}_{\mathrm{w}}$ & $\begin{array}{l}\text { Mean } \\
(\mathrm{SD})\end{array}$ & $\mathrm{SEM}_{\mathrm{w}}$ & $\begin{array}{l}\text { Mean } \\
\text { (SD) }\end{array}$ & $\mathrm{SEM}_{\mathrm{w}}$ & $\begin{array}{l}\text { Mean } \\
\text { (SD) }\end{array}$ & $\mathrm{SEM}_{\mathrm{w}}$ & $\begin{array}{l}\text { Mean } \\
\text { (SD) }\end{array}$ & $\mathrm{EM}_{\mathrm{w}}$ \\
\hline ithin sess & mean $(\mathrm{SD})$ and $\mathrm{st}$ & star & eeasuren & en & $\mathrm{SEM}_{\mathrm{w}}$ ) of the $\mathrm{t}$ & iporal $p_{\text {. }}$ & neters & int an & ngles at & ie sel & $\mathrm{d}$ upper lin & ks. & & & & & & \\
\hline Temporal & & & & & & & & & & & & & & & & & & \\
\hline Duration & $0.88(0.13)$ & 0.0 & $0.98(0.16)$ & $0.0 \mathrm{~s}$ & $0.90(0.16)$ & 0.06 & $0.99(0.18)$ & 0.08 & $0.97(0.16)$ & $0.0 \mathrm{~s}$ & $1.00(0.16)$ & 0.07 & $0.78(0.15)$ & 0.06 & $0.88(0.16)$ & 0.10 & $0.79(0.12)$ & 0.07 \\
\hline Speed & $0.52(0.09)$ & 0.0 & $0.63(0.15)$ & 0.06 & $0.63(0.13)$ & 0.04 & $0.49(0.11)$ & 0.04 & $0.47(0.10)$ & 0.0 & $0.46(0.09)$ & 0.04 & $0.49(0.10)$ & 0.04 & $0.76(0.16)$ & 0.07 & $0.52(0.12)$ & 0.04 \\
\hline Trunk & & & & & & & & & & & & & & & & & & \\
\hline Flexion & $0.9(6.0)$ & 1.6 & $-0.3(6.8)$ & 2.3 & $0.2(8.3)$ & 2.4 & $2.7(7.0)$ & 2.1 & $3.9(5.8)$ & 2.0 & $3.3(6.6)$ & 2.4 & $1.4(6.7)$ & 2.5 & $-4.4(5.8)$ & 1.8 & $-1.3(7.0)$ & 2.5 \\
\hline Lateral flexion & $\mathrm{n} \quad-5.4(3.0)$ & 1.2 & $-0.5(5.2)$ & 2.2 & $-7.3(2.8)$ & 1.4 & $-6.2(4.3)$ & 2.2 & $-6.5(3.4)$ & 1.6 & $-6.0(3.6)$ & 1.6 & $0.8(3.0)$ & 1.5 & $-4.6(2.3)$ & 1.8 & $-0.8(2.8)$ & 2.0 \\
\hline Rotation & $8.6(4.3)$ & 2.7 & $-12.9(9.3)$ & 4.3 & $9.3(5.9)$ & 3.0 & $11.7(5.6)$ & 3.3 & $12.6(5.8)$ & 2.6 & $12.6(5.5)$ & 3.6 & $-0.5(8.0)$ & 3.6 & $-1.5(7.9)$ & 2.5 & $5.5(7.2)$ & 2.8 \\
\hline Scapula & & & & & & & & & & & & & & & & & & \\
\hline Pro-retract & $47.4(7.2)$ & 2.9 & $24.4(10.8)$ & 3.8 & $48.0(8.9)$ & 3.1 & $47.4(6.7)$ & 2.3 & $46.2(8.3)$ & 2.8 & $47.1(7.1)$ & 2.5 & $33.9(6.7)$ & 2.8 & $24.4(8.4)$ & 3.9 & $47.7(7.7)$ & 2.2 \\
\hline Med-lat rot & $-23.2(5.4)$ & 2.5 & $-22.1(5.8)$ & 2.5 & $-28.0(7.4)$ & 2.8 & $-22.1(6.0)$ & 2.9 & $-20.4(5.4)$ & 2.8 & $-19.9(5.2)$ & 3.3 & $-14.0(7.4)$ & 2.8 & $-36.3(5.1)$ & 2.4 & $-16.6(8.6)$ & 4.1 \\
\hline Tilting & $1.2(5.7)$ & 2.5 & $2.9(5.8)$ & 3.2 & $3.8(7.0)$ & 3.1 & $2.1(6.0)$ & 2.1 & $4.3(4.9)$ & 1.9 & $2.0(5.2)$ & 2.3 & $-5.6(4.8)$ & 2.0 & $11.3(6.9)$ & 3.0 & $-1.1(6.0)$ & 1.6 \\
\hline Shoulder & & & & & & & & & & & & & & & & & & \\
\hline Elev plane & $74.0(10.9)$ & 3.6 & $21.3(7.3)$ & 4.6 & $71.6(12.2)$ & 4.1 & $71.9(10.0)$ & 3.1 & $75.8(9.3)$ & 3.1 & $70.6(10.4)$ & 3.9 & $78.6(9.3)$ & 5.8 & $52.6(15.1)$ & 5.8 & $97.5(6.6)$ & 3.6 \\
\hline Elevation & $-86.9(8.6)$ & 3.6 & $-84.0(6.3)$ & 3.9 & $-102.1(6.1)$ & 3.3 & $-88.8(7.1)$ & 2.6 & $-85.5(7.9)$ & 2.8 & $-86.7(7.0)$ & 3.9 & $-45.6(11.1)$ & 4.0 & $-108.5(9.5)$ & 5.2 & $-42.9(9.0)$ & 3.9 \\
\hline Rotation & $-42.8(12.1)$ & 3.6 & $-40.0(11.6)$ & 4.4 & $-41.0(11.7)$ & 4.2 & $-42.7(11.0)$ & 3.5 & $-54.3(9.4)$ & 3.4 & $-42.4(10.8)$ & 3.9 & $-43.9(11.6)$ & 3.6 & $-53.8(9.8)$ & 3.2 & $-31.5(13.3)$ & 3.4 \\
\hline Elbow & & & & & & & & & & & & & & & & & & \\
\hline Flexion & $27.4(14.8)$ & 5.3 & $13.4(9.8)$ & 6.1 & $21.3(9.2)$ & 4.1 & $26.0(13.0)$ & 5.5 & $26.1(11.4)$ & 4.9 & $27.5(12.0)$ & 6.1 & $135.4(5.3)$ & 2.3 & $106.9(7.4)$ & 3.4 & $103.0(5.9)$ & 3.7 \\
\hline Pro-sup & $124.7(11.2)$ & 3.1 & $120.4(14.0)$ & 4.7 & $121.8(12.2)$ & 3.9 & $124.1(11.1)$ & 3.7 & $85.0(12.5)$ & 3.1 & $129.8(11.8)$ & 2.3 & $77.4(12.6)$ & 5.1 & $76.8(13.8)$ & 3.3 & $88.7(10.2)$ & 4.2 \\
\hline Wrist & & & & & & & & & & & & & & & & & & \\
\hline Flexion & $5.5(7.1)$ & 6.2 & $3.2(8.4)$ & 6.4 & $5.4(8.1)$ & 6.7 & $-5.9(7.5)$ & 5.7 & $-10.2(13.2)$ & 8.5 & $-20.6(6.1)$ & 6.0 & $3.8(10.8)$ & 8.2 & $21.0(14.2)$ & 7.4 & $22.8(13.5)$ & 5.1 \\
\hline Deviation & $-1.9(6.8)$ & 4.1 & $-1.2(5.8)$ & 5.2 & $-2.2(6.8)$ & 4.9 & $-3.9(7.3)$ & 5.3 & $-0.3(9.4)$ & 5.5 & $-2.7(5.8)$ & 4.3 & $-14.3(14.9)$ & 4.6 & $-22.4(11.0)$ & 4.7 & $-10.2(11.7)$ & 5.8 \\
\hline & Reach forwards & & Reach sideways & & Reach upwards & & $\begin{array}{l}\text { Reach to grasp } \\
\text { spherically }\end{array}$ & & $\begin{array}{l}\text { Reach to grasp } \\
\text { vertically }\end{array}$ & & $\begin{array}{l}\text { Reach to grasp } \\
\text { horizontally }\end{array}$ & & Hand to mouth & & Hand to head & & Hand to should & \\
\hline & $\begin{array}{l}\text { Mean } \\
\text { (SD) }\end{array}$ & $\mathrm{SEM}_{\mathrm{b}}$ & $\begin{array}{l}\text { Mean } \\
\text { (SD) }\end{array}$ & $\mathrm{SEM}_{\mathrm{b}}$ & $\begin{array}{l}\text { Mean } \\
\text { (SD) }\end{array}$ & $\mathrm{SEM}_{\mathrm{b}}$ & $\begin{array}{l}\text { Mean } \\
\text { (SD) }\end{array}$ & $\mathrm{SEM}_{\mathrm{b}}$ & $\begin{array}{l}\text { Mean } \\
(\mathrm{SD})\end{array}$ & $\mathrm{SEM}_{\mathrm{b}}$ & $\begin{array}{l}\text { Mean } \\
\text { (SD) }\end{array}$ & $\mathrm{SEM}_{\mathrm{b}}$ & $\begin{array}{l}\text { Mean } \\
\text { (SD) }\end{array}$ & $\mathrm{SEM}_{\mathrm{b}}$ & $\begin{array}{l}\text { Mean } \\
\text { (SD) }\end{array}$ & $\mathrm{SEM}_{\mathrm{b}}$ & $\begin{array}{l}\text { Mean } \\
\text { (SD) }\end{array}$ & $\mathrm{EM}_{\mathrm{b}}$ \\
\hline $\begin{array}{l}\text { B. Between } \mathrm{s} \\
\text { Temporal }\end{array}$ & on mean (SD) and & $\mathrm{ds}$ & $\mathrm{fm}$ & Ire & the & mporal pa & arameters, and the & $\mathrm{h}$ & angles at PTA for $\mathrm{t}$ & the sele & ected unner limb t & & & & & & & \\
\hline Duration & $0.87(0.12)$ & 0.0 & $0.94(0.14)$ & 0.04 & $0.88(0.13)$ & 0.08 & $0.95(0.15)$ & 0.08 & $0.94(0.13)$ & 0.05 & $0.96(0.13$ & 0.04 & $0.75(0.12)$ & 0.06 & $0.85(0.12)$ & 0.04 & $0.77(0.10)$ & 0.04 \\
\hline Speed & $0.53(0.09)$ & 0.0 & $0.66(0.14)$ & 0.03 & $0.64(0.12)$ & 0.05 & $0.50(0.10)$ & 0.04 & $0.48(0.10)$ & 0.03 & $0.48(0.09)$ & 0.02 & $0.51(0.09)$ & 0.05 & $0.77(0.12)$ & 0.07 & $0.54(0.11)$ & 0.06 \\
\hline Trunk & & & & & & & & & & & & & & & & & & \\
\hline Flexion & $-0.5(4.8)$ & 4.2 & $-1.6(6.6)$ & 4.2 & $-2.5(7.2)$ & 5.2 & $1.0(5.8)$ & 5.0 & $1.6(5.4)$ & 5.1 & $2.1(5.4)$ & 4.8 & $-0.3(5.6)$ & 4.9 & $-5.9(5.6)$ & 4.7 & $-3.2(6.4)$ & 6.2 \\
\hline Lateral flexion & $\mathrm{n} \quad-5.5(2.1)$ & 2.0 & $-1.2(4.2)$ & 3.0 & $-7.1(2.1)$ & 1.4 & $-6.1(3.2)$ & 2.4 & $-6.2(2.8)$ & 1.9 & $-6.2(2.9)$ & 2.3 & $0.4(2.5)$ & 1.0 & $-4.4(2.5)$ & 1.8 & $-1.0(2.3)$ & 1.2 \\
\hline Rotation & $9.2(3.6)$ & 2.8 & $-13.2(6.9)$ & 4.8 & $9.6(4.7)$ & 3.9 & $12.5(5.0)$ & 4.1 & $12.6(5.1)$ & 3.9 & $13.5(5.6)$ & 3.7 & $-1.9(7.5)$ & 5.3 & $-3.5(6.7)$ & 5.3 & $5.4(7.7)$ & 7.0 \\
\hline Scapula & & & & & & & & & & & & & & & & & & \\
\hline Pro-retract & $47.7(8.0)$ & 7.1 & $25.1(10.2)$ & 7.2 & $47.7(8.9)$ & 7.1 & $47.6(7.0)$ & 6.0 & $47.2(7.5)$ & 6.9 & $47.5(7.0)$ & 5.8 & $34.0(6.2)$ & 6.2 & $22.8(7.8)$ & 7.2 & $47.8(9.1)$ & 4.8 \\
\hline Med-lat rot & $-22.9(4.8)$ & 3.2 & $-21.1(5.0)$ & 4.8 & $-27.0(6.0)$ & 5.2 & $-20.8(5.1)$ & 4.1 & $-19.2(4.7)$ & 3.7 & $-19.4(4.1)$ & 3.5 & $-14.0(7.0)$ & 3.1 & $-35.0(5.3)$ & 4.1 & $-15.4(7.7)$ & 5.7 \\
\hline Tilting & $1.7(5.3)$ & 3.3 & $2.0(6.1)$ & 3.1 & $4.3(6.7)$ & 3.3 & $2.4(6.5)$ & 3.6 & $4.1(5.3)$ & 3.1 & $2.6(6.0)$ & 3.7 & $-5.2(5.3)$ & 3.7 & $11.6(6.3)$ & 2.2 & $-1.5(5.8)$ & 4.1 \\
\hline Shoulder & & & & & & & & & & & & & & & & & & \\
\hline Elev plane & $75.9(9.9)$ & 6.0 & $22.1(5.4)$ & 2.7 & $73.0(10.6)$ & 4.7 & $72.3(9.0)$ & 6.0 & $77.1(8.3)$ & 4.9 & $71.7(8.8)$ & 4.9 & $78.7(10.2)$ & 6.6 & $52.3(14.0)$ & 9.9 & $99.2(5.3)$ & 3.2 \\
\hline Elevation & $-86.7(7.3)$ & 3.4 & $-83.0(5.8)$ & 3.0 & $-99.9(6.1)$ & 3.2 & $-87.3(7.1)$ & 3.0 & $-84.0(8.9)$ & 4.2 & $-86.1(6.3)$ & 2.9 & $-46.0(9.5)$ & 3.7 & $-106.7(8.6)$ & 5.1 & $-42.3(8.2)$ & 5.9 \\
\hline Rotation & $-40.1(10.8)$ & 7.0 & $-36.1(10.4)$ & 9.0 & $-38.3(10.4)$ & 7.4 & $-39.6(10.4)$ & 7.6 & $-50.7(10.3)$ & 7.3 & $-39.8(9.8)$ & 7.0 & $-41.8(10.0)$ & 6.9 & $-51.7(9.6)$ & 7.4 & $-30.0(10.7)$ & 8.1 \\
\hline Elbow & & & & & & & & & & & & & & & & & & \\
\hline Flexion & $26.0(12.7)$ & 5.1 & $14.2(10.0)$ & 5.6 & $21.8(10.3)$ & 5.4 & $26.3(12.9)$ & 3.6 & $25.9(11.3)$ & 4.0 & $27.7(12.2)$ & 4.3 & $135.8(5.8)$ & 2.1 & $108.0(6.5)$ & 2.4 & $103.7(4.6)$ & 2.7 \\
\hline Pro-sup & $120.9(11.2)$ & 8.0 & $116.6(12.7)$ & 9.2 & $117.9(11.3)$ & 7.5 & $120.4(10.9)$ & 6.7 & $81.3(11.3)$ & 7.5 & $125.4(12.2)$ & 7.9 & $78.5(10.6)$ & 8.8 & $78.3(12.3)$ & 7.6 & $87.1(10.5)$ & 5.7 \\
\hline Wrist & & & & & & & & & & & & & & & & & & \\
\hline Flexion & $5.5(4.4)$ & 4.4 & $4.7(6.2)$ & 4.9 & $6.7(4.6)$ & 4.7 & $-7.2(5.3)$ & 5.8 & $-12.0(9.8)$ & 8.6 & $-19.6(5.0)$ & 4.4 & $5.2(11.4)$ & 6.6 & $24.4(10.5)$ & 5.7 & $23.6(10.4)$ & 6.9 \\
\hline Deviation & $0.8(6.2)$ & 3.6 & $1.9(5.3)$ & 4.6 & $-0.3(5.6)$ & 4.4 & $-2.7(6.7)$ & 3.9 & $0.9(8.6)$ & 4.1 & $-2.8(5.3)$ & 4.3 & $-13.4(14.8)$ & 2.8 & $-23.1(9.2)$ & 4.9 & $-8.1(9.3)$ & 4.6 \\
\hline
\end{tabular}




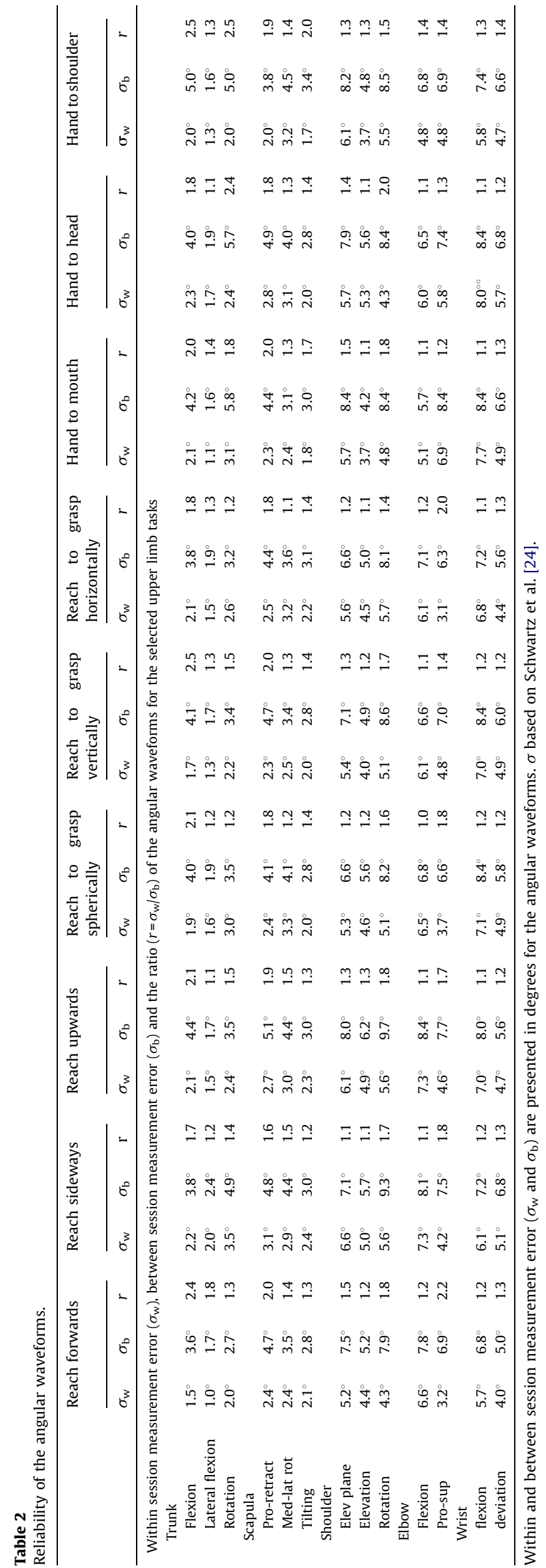

This study demonstrated that the proposed protocol can be used as a reliable tool for the evaluation of UL movements in TDC. Still, results are based on a relatively small group of 10 TDC, with a wide age range. Proper age-related reference data of UL joint kinematics for the selected tasks can only be established through the assessment of a larger group of TDC. This will also permit to further explore the influence of age on the maturation of joint kinematics. Although previous studies have already shown that several aspects of reaching, e.g. smoothness and hand trajectories, vastly improve until the age of 10-12 years [29,30], literature on the maturation of joint kinematics is scarce. Knowledge on how TDC perform the tasks will allow the comparison to children with UL movement pathology, e.g. HCP. To comprehend the true value of the developed procedure as a clinical tool, its reliability should be confirmed in children with HCP. Further study on the comparison between TDC and children with HCP, based on larger study samples, and a more in-depth analysis on the relation between UL kinematics and clinical parameters, will help to construct a wellfounded final task-selection, depending on the clinical question.

\section{Acknowledgement}

Ellen Jaspers received a PhD fellowship of the Research Foundation-Flanders (FWO), Belgium.

\section{Conflict of interest statement}

The authors report no financial or personal relationships with other people or organizations that could inappropriately influence their work.

\section{Appendix A. Supplementary data}

Supplementary data associated with this article can be found, in the online version, at doi:10.1016/j.gaitpost.2010.11.021.

\section{References}

[1] Coluccini M, Maini ES, Martelloni C, Sgandurra G, Cioni G. Kinematic characterization of functional reach to grasp in normal and in motor disabled children. Gait Posture 2007;25:493-501.

[2] Fitoussi F, Maurel N, Diop A, Laasel EM, Ilharreborde B, Presedo A, et al. Upper extremity kinematics analysis in obstetrical brachial plexus palsy. Orthop Traum Surg Res 2009;95:336-42.

[3] Jaspers E, Desloovere K, Bruyninckx H, Molenaers G, Klingels K, Feys H. Review of quantitative measurements of upper limb movements in hemiplegic cerebral palsy. Gait Posture 2009;30:395-404.

[4] Kontaxis A, Cutti AG, Johnson GR, Veeger HE. A framework for the definition of standardized protocols for measuring upper-extremity kinematics. Clin Biomech (Bristol Avon) 2009;24:246-53.

[5] Schmidt R, Disselhorst-Klug C, Silny J, Rau G. A marker-based measurement procedure for unconstrained wrist and elbow motions. J Biomech 1999;32:615-21.

[6] Williams S, Schmidt R, Disselhorst-Klug C, Rau G. An upper body model for the kinematical analysis of the joint chain of the human arm. J Biomech 2006;39:2419-29.

[7] Rab G, Petuskey K, Bagley A. A method for determination of upper extremity kinematics. Gait Posture 2002;15:113-9.

[8] Carpinella I, Mazzoleni P, Rabuffetti M, Throsen R, Ferrarin M. Experimental protocol for the kinematic analysis of the hand: definition and repeatability. Gait Posture 2006;23:445-54.

[9] Wu G, van der Helm FCT, Veeger HEJ, Makhsous M, Van Roy P, Anglin C, et al. ISB recommendation on definitions of joint coordinate systems of various joints for the reporting of human joint motion-part II: shoulder, elbow, wrist and hand. J Biomech 2005;38:981-92.

[10] Petuskey K, Bagley A, Abdala E, James MA, Rab G. Upper extremity kinematics during functional activities: three-dimensional studies in a normal pediatric population. Gait Posture 2007;25:573-9.

[11] Butler EE, Lad AL, Louie SA, LaMont LE, Wong W, Rose J. Three-dimensional kinematics of the upper limb during a Reach and Grasp Cycle for children. Gait Posture 2010;32:72-7.

[12] Reid S, Elliott C, Alderson J, Lloyd D, Elliott B. Repeatability of upper limb kinematics for children with and without cerebral palsy. Gait Posture 2010;32:10-7.

[13] Mottram SL. Dynamic stability of the scapula. Man Ther 1997;2:123-31. 
[14] Randall M, Johnson L, Reddihough D. The Melbourne assessment of unilateral upper limb function: test administration manual. Melbourne: Royal Children's Hospital; 1999.

[15] van Andel CJ, Wolterbeek N, Doorenbosch CA, Veeger DH, Harlaar J. Complete 3D kinematics of upper extremity functional tasks. Gait Posture 2008;27: 120-7.

[16] Söderkvist I, Wedin PA. Determining the movements of the skeleton using well-configured markers. J Biomech 1993;26:1473-7.

[17] Capozzo A, Catani F, Della Croce U, Leardini A. Position and orientation in space of bones during movement: anatomical frame definition and determination. Clin Biomech 1995;10:171-8.

[18] Meskers CGM, van der Helm FCT, Rozendaal LA, Rozing PM. In vivo estimation of the glenohumeral joint rotation center from scapular bony landmarks by linear regression. J Biomech 1998;31:93-6.

[19] van Sint Jan S. Color atlas of skeletal landmark definitions: guidelines for reproducible manual and virtual palpation. Belgium: Churchill Livingstone; 2007.

[20] Woltring HJ. Smoothing and differentiation techniques applied to 3-D data. In: Allard P, Stokes IAF, Blanchi J-P, editors. Three-dimensional analysis of human movement. Champaign: Human Kinetics Publishers; 1995.

[21] Weir JP. Quantifying test-retest reliability using the intraclass correlation coefficient and the SEM. J Strength Cond Res 2005;19:231-40.
[22] Katz JN, Larson MG, Phillips CB, Fossel AH, Liang MH. Comparative measurement sensitivity of short and longer health status instruments. Med Care 1992;30:917-25

[23] Kadaba MP, Ramakrishnan HK, Wootten ME, Gainey J, Gorton G, Cochran GV. Repeatability of kinematic, kinetic, and electromyographic data in normal adult gait. J Orthop Res 1989;7:849-60.

[24] Schwartz MH, Trost JP, Wervey RA. Measurement and management of errors in quantitative gait data. Gait Posture 2004;20:196-203.

[25] McGinley JL, Baker R, Wolfe R, Morris ME. The reliability of three-dimensional kinematic gait measurements: a systematic review. Gait Posture 2009;29:360-9.

[26] Senk M, Chèze L. Rotation sequence as an important factor in shoulder kinematics. Clin Biomech 2006;21:S3-8.

[27] Cutti AG, Giovanardi A, Rocchi L, Davalli A, Sacchetti R. Ambulatory measurement of shoulder and elbow kinematics through inertial and magnetic sensors. Med Biol Eng Comput 2008;46:169-78.

[28] Wolf SI, Fradet L, Retig O. Conjunct rotation: Codman's paradox revisited. Med Biol Eng Comput 2009;47:551-6.

[29] Berthier NE, Keen R. Development of reaching in infancy. Exp Brain Res 2006;169:507-18.

[30] Schneiberg S, Sveistrup H, McFadyen B, McKinley P, Levin MF. The development of coordination for reach-to-grasp movements in children. Exp Brain Res 2002;146:142-54 